Perspectiva Geográfica

ISSN 0123-3769

Vol.13 • Págs. $13-26$

Diciembre 2008

\title{
Identificación de conflictos de uso de suelo en rondas hídricas: herramienta para manejo ambiental.Caso de estudio municipio de Paipa*
}

\author{
Land use conflict identification in watersheds: an \\ environmental management tool. Case study in the Paipa \\ county.
}

\section{Omar Javier Daza Leguizamón** Rigaud Sanabria Marin***}

\section{Resumen}

La conservación y recuperación de las rondas hídricas, a partir de la identificación de áreas de intervención prioritaria, son aspectos necesarios para asegurar la disponibilidad y sostenibilidad del recurso hídrico. En este estudio, se propone una metodología que, mediante la utilización de sistemas de información geográfica einformación secundaría de los planes de ordenamiento territorial, facilite la identificación y cuantificación de rondas hídricas expuestas a la potencial degradación causada por los conflictos de uso del suelo presentes, y así dar criterios para la intervención y manejo ambiental de estas áreas. En el municipio de Paipa, se encontró que de las 3.420 hectáreas de ronda hídrica, el $68 \%$ se encuentra en zonas de conflictos de uso del suelo alto y medio, viéndose afectadas, principalmente, las rondas de las veredas M edios y M arcura.

Palabras clave: Boyacá, Conflictos de uso de la tierra; Cuenca hidrográfica; Ordenamiento Territorial; Paipa; sistemas de información geográfica.

\footnotetext{
Trabajo de Investigación del Grupo de Investigación en Geomática y Ambi ente (GIGA).

* Joven Investigador, Grupo de Investigación en Geomática y Ambiente, Uni versidad Pedagógica y Tecnol ógica de Colombia.

Correo: ki anosla@gmail.com

*** Profesor asociado, Escuel a de Ingeni ería Civil, Uni versidad Pedagógica y Tecnológica de Col ombia.

Correo: sanabriarigaud@ahoo.com
} 


\section{Abstract}

The conservation and restoration of watersheds, from the identification of areas of priority intervention, is necessary to attain the availability and sustainability of the water resource. In this study, we propose a methodology based on geographic information systems and secondary data from space plans. The methodology enables the identification and quantification of watersheds prone to degradation as a result of land use conflicts. The purpose is to bestow criteria for environmental management. In the borough of Paipa, Boyacá, Colombia we find that of the 3.420 ha of the watershed, $68 \%$ of them are in high of medium land use conflicts. Furthermore, the corridors of M arcura and M edios are the most affected.

Key words: Boyacá; geographic information systems; land use conflicts; Paipa; space planning; watersheds. 


\section{Introducción}

Las rondas hídricas, o áreas de transición entre el medio netamente acuático y el terrestre (Bentrup y Kellerman, 2004; Jarro, 2004), son zonas de gran importancia ambiental, por sus funciones de captación y remoción de sedimentos de la escorrentía, estabilización de taludes, captación y remoción de contaminantes, almacenamiento de aguas de inundación, regulación de la temperatura del agua, provisión de hábitat para organismos terrestres, recreación y educación (D epartament of Biological and Agricultural Engineering, 2007; Wender, 1999).

Dada la interrelación entre la corriente de agua y la ronda hídrica, la alteración de alguna de las dos puede afectar los procesos comunes a ellas (Jarro, 2004; Departament of Biological and Agricultural Engineering, 2007), por lo cual el cambio de la vegetación nativa en estos corredores influye en la calidad y cantidad del recurso hídrico. Como caso particular, en el área rural del departamento de Boyacá, es común encontrar que la cobertura natural de las riberas ha sido cambiada con el fin de aprovechar la madera de bosques de ripario y ampliar las áreas para cultivo y pastoreo, usos del suelo que son los principales responsables de la degradación de las rondas (Corpoboyacá e Hidrogeología y Geotecnia Ambiental, 2004). Esta situación pone en evidencia la necesidad de adelantar estudios con el fin de identificar áreas en estado crítico de afectación, y tomar medidas correctivas que mitiguen los actuales impactos ambientales negativos.

A pesar de que la normatividad colombiana considera las rondas hídricas como bienes inalienables e imprescindibles del Estado (Presidencia de la República de Colombia, 1974) y como áreas de conservación y preservación del recurso hídrico (Presidencia de la República de Colombia, 1998), dentro del departamento de Boyacá, y en general en el país, no se les ha dado la importancia que demandan.

Un referente para la formulación, ejecución y seguimiento de proyectos de restauración ecológica, en rondas y nacederos de agua para Colombia, es la guía técnica elaborada para tal fin, en la ciudad de Bogotá, D.C. (Jarro, 2004). Los Sistemas de Información Geográfica, SIG, se utilizan en diversas metodologías para la delimitación de rondas hídricas, cálculo de pendientes, estrategias de priorización con fines de recuperación y evaluación de impactos causados por usos agropecuarios.

En la cuenca de Cumberland en Tennesse, Estados Unidos, se realizó la medición de la pendiente de las rondas hídricas mediante el empleo de modelos digitales de elevación, estableciendo como límite de los corredores las áreas no intervenidas a lo ancho de la corriente (Lamoine y Smith, 2006). Williams et al. (2003) emplean herramientas de SIG y modelos digitales de elevación para la delimitación de rondas hídricas variables, en función de la pendiente de 
la ribera. Bentrup y Kellerman (2004) plantean una metodología para la ubicación de zonas de recuperación en rondas hídricas, variando el ancho de corredor según el orden de la corriente, a partir del análisis de la conectividad de remanentes de vegetación nativa. Dentro de esta última metodología, se consideraron actividades generales, como la identificación de remanentes, la localización de áreas dentro del corredor para la implementación de la reconexión de remanentes y la ubicación de barreras viales que limitaran la conectividad. Redal et al. (2005) realizaron la evaluación del impacto ambiental de las actividades productivas en el corredor biológico Alexander Skutch, Costa Rica, sobre una zona de protección de $100 \mathrm{~m}$, a cada lado de las corrientes.

El presente estudio, realizado en el municipio de Paipa, propone una metodología que, mediante sistemas de información geográfica, facilite identificar los conflictos de uso del suelo que afectan las rondas y corrientes hídricas, con el fin de establecer un criterio para la intervención y manejo ambiental de este tipo de ecosistemas. Se parte de la zonificación de conflictos de uso del suelo, considerando que una alta conflictividad en el uso de la tierra está asociada con una alta degradación ambiental (Instituto Geográfico Agustín Codazzi, 2000). Se consideran dos enfoques: el primero identifica las áreas con conflictos de uso alto y medio dentro del área de ronda; el segundo, cuantifica la longitud total de las corrientes asociadas con la alta conflictividad de uso, para realizar una valoración y permitir priorizar la reforestación de zonas de ronda hídrica.

\section{M ateriales y M etodología}

El estudio piloto fue realizado en el municipio de Paipa, ubicado en la parte noroccidental del departamento de Boyacá, a $2.525 \mathrm{msnm}$, el cual cuenta con un área de 30.592 ha, divididas en 38 veredas y la zona urbana. Las principales corrientes que atraviesan el municipio son las quebradas Valencí, El Rosal, El Salitre y el Río Chicamocha; este último, presenta la mayor parte del área de su cuenca y su nacimiento en el departamento de Boyacá. Los principales usos del suelo son la agricultura y la ganadería, con el $43 \%$ del área total municipal (Instituto Geográfico Agustín Codazzi, 2000).

Los conflictos de uso del suelo se presentan cuando hay discrepancia entre el uso que debería tener el suelo, de acuerdo con su oferta ambiental, y aquel al que está expuesto por las actividades humanas (IGAC y Corpoica, 2002). Para el municipio de Paipa, la zonificación de conflictos de uso del suelo permite identificar zonas prioritarias de intervención, que se presentan principalmente por la ocupación de tierras con potencial forestal actualmente utilizadas en agricultura o ganadería; situación que degrada los ecosistemas y causa impactos ambientales negativos (Naizaque, 2001), a través de la 
contaminación con material orgánico y agroquímicos (Redal et al, 2005).

Cuando este tipo de conflictos se presenta, en cercanías a una corriente hídrica, se puede incrementar el riesgo de que los contaminantes lleguen al cuerpo de agua y lo afecten. Además, un uso del suelo diferente al potencial pone en grave peligro la calidad del recurso suelo (Naizaque, 2001; IGAC y Corpoica, 2002), pudiéndose llegar a situaciones en las que una avanzada erosión dificulte las acciones de reforestación y recuperación ambiental de la ronda, al reducirse la fertilidad y la capacidad de almacenamiento de agua del suelo (Gonzáles, 2005).

La información fuente, tomada del Plan de Ordenamiento Territorial, POT, de Paipa, corresponde a la zonificación de conflictos de uso del suelo y a la red hidrográfica. La información tomada del POT, se encuentra en formato digital compatible con el software AcrG IS y en sistema de coordenadas proyectadas con datum Bogotá. Para la zonificación de conflictos de uso del suelo en el POT de Paipa, se definen zonas de conflictos de uso alto, medio, bajo y equilibrio, así como zonas en conflicto por el crecimiento urbano.

El conflicto alto, que cubre el $23 \%$ del municipio, se presenta en tierras con aptitudes para conservación, recuperación y turismo ecológico que son utilizadas para la ganadería extensiva y agricultura tradicional. El conflicto medio, con un $49 \%$ del área municipal, se presenta en suelos con aptitud para agricultura tradicional y recuperación, utilizados para ganadería extensiva y extracción-producción. En el conflicto bajo, presente en un $17 \%$ del territorio, se encuentra principalmente relaciones de aptitud para ganadería semi-intensiva y uso en ganadería extensiva y agricultura tradicional, aptitud para agricultura semimecanizada y utilización en ganadería extensiva, y aptitud para turismo cultural y uso de extracción y producción. En las áreas en equilibrio, 9,5\% del municipio, corresponde el uso con la aptitud del suelo en tierras para ganadería semi-intensiva y ganadería extensiva. Finalmente, el crecimiento urbano produce conflictos en las áreas que rodean la zona urbana del municipio y corresponde a un $1,5 \%$ del área total (Instituto Geográfico Agustín Codazzi, 2000).

Para identificar la conflictividad de uso del suelo en las rondas hídricas, se abordó el problema desde dos enfoques: primero, se determinaron las áreas de conflicto de uso del suelo dentro de las áreas de ronda hídrica (Figura 1a), con el fin de localizar los sitios de conflictos medio y alto. En un segundo enfoque, se analizó la longitud de las corrientes, de acuerdo con su paso por las zonas de conflictividad de uso (Figura 1b), con el fin de identificar los lugares donde el uso inadecuado del suelo esté afectando directamente la corriente de agua. Con el fin de orientar la toma de decisiones, de acuerdo con la prioridad de los corredores, se caracterizaron las rondas para cada vereda y para las principales corrientes hídricas del municipio. 


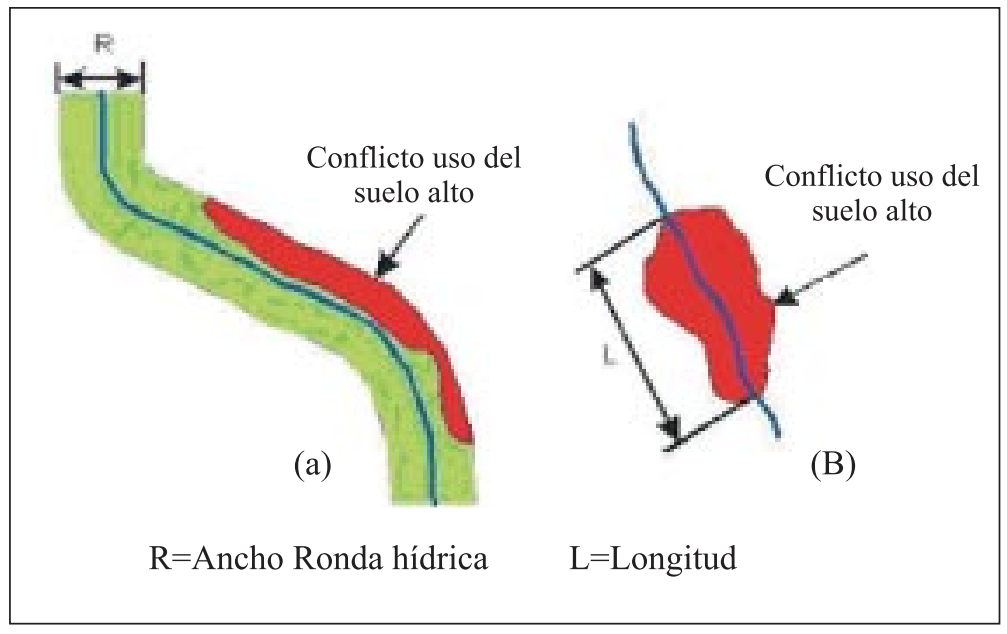

Figura 1. Conflictos de uso del suelo altos, asociados con la ronda hídrica (a) y con la corriente de agua (b) Fuente: elaboración propia

La implementación de la metodología se hizo con el software ArcG is 9.0 (Environmental Systems Research Institute, 2004), siguiendo el modelo matemático y cartográfico representado en la figura 2 , en el cual, se presenta la secuencia de operaciones de análisis espacial, orientadas a resolver la problemática identificada (Sanabria, 1999). Inicialmente, se realizó la delimitación de las rondas hídricas, la cual corresponde a un corredor de 30 $\mathrm{m}$, a cada lado de la corriente, de acuerdo con lo establecido en la normatividad colombiana (Presidencia de la República de Colombia, 1974); de este layer, se calculó el área total de rondas hídricas en el municipio. Para la localización de los conflictos de uso del suelo, dentro de las rondas establecidas, se realizó una sobreposición de estas dos coberturas, omitiendo las áreas de conflicto de uso del suelo que estuvieran por fuera de las rondas; de este nuevo layer, se calcularon las áreas para cada uno de los conflictos de uso del suelo dentro de los corredores, con sus respectivos porcentajes, para estimar el estado general de estas áreas dentro del municipio, dentro de cada vereda y para las corriente principales. De otro lado, mediante la sobreposición de la red hidrográfica y la zonificación de conflictos, se localizaron y contabilizaron la longitud de las corrientes que atraviesan los diferentes tipos de conflictos de uso. 


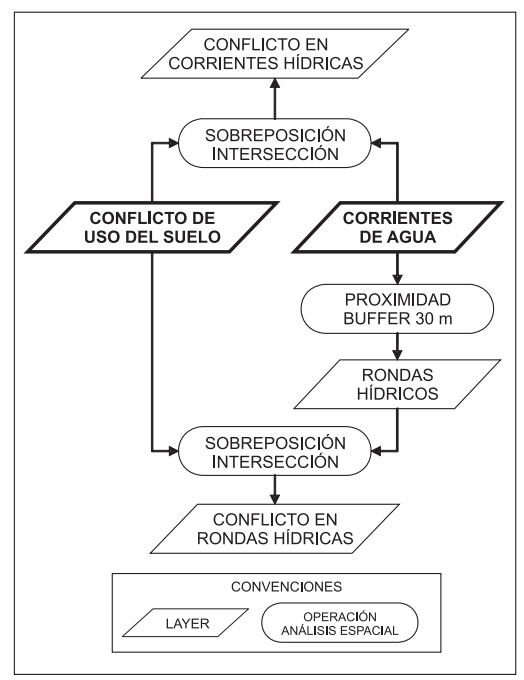

Figura 2. Modelo cartográfico de conflicto de uso en rondas y corrientes hídricas.

Fuente: elaboración propia

Con el fin de evaluar la conflictividad de las rondas hídricas y corrientes para cada vereda, se propone el indicador Grado de Conflicto de Uso del suelo (Ecuación 1), calculado a partir de las áreas de cada conflicto de uso del suelo presente en las rondas hídricas. El indicador compara las áreas de conflictos, consideradas como las causantes de mayores impactos ambientales negativos (alto, medio y por crecimiento urbano), frente a las que causan degradación baja o nula del suelo (bajo y equilibrio).

(1) $G C=\frac{(E+B) \cdot(A+M+Z)}{A V}$

En donde:

E es el área en la vereda sin conflicto de uso del suelo

B es el área en la vereda con conflicto de uso del suelo bajo

$M$ es el área en la vereda con conflicto de uso del suelo medio

A es el área en la vereda con conflicto de uso del suelo alto

$Z$ es el área en la vereda con conflicto de uso causado por el crecimiento urbano

$A v$ es el área total de ronda en la vereda analizada.

Los valores posibles para el indicador varían de -1 a 1 , correspondiendo el valor de -1 para las veredas que tienen en su totalidad conflictos de uso medio, alto y por crecimiento urbano; el valor de 1 a las veredas en las que la totalidad de las zonas de protección se encuentran en equilibrio o tienen un conflicto de uso del suelo bajo. Un valor igual a cero, se da en las veredas en las que los conflictos 
alto, medio y por crecimiento urbano, son iguales a las zonas en equilibrio y conflicto bajo.

\section{Resultados}

El mapa resultado localiza cada una de las corrientes dentro del municipio, su ronda y los conflictos por uso del suelo presentes (el mapa completo no se presenta en este artículo). En la Figura 3 , se puede ver la alta predominancia de usos inadecuados del suelo que afectan el recurso hídrico en las quebradas: El Campanario, El Chuscal y El Chorro; situación que se generaliza para todo el municipio. Así, se encontró que la alta y media conflictividad predominan en la parte norte del municipio y afectan principalmente la cuenca del río Palermo. El conflicto de uso bajo y las áreas en equilibrio, corresponden a las rondas de menor altura del municipio (menores a 2.800 $\mathrm{m})$, donde las aptitudes del suelo están orientadas hacia actividades productivas.

Del área total del municipio, 3.420 ha $(11 \%)$ corresponden a zonas de rondas hídricas, de las cuales $68 \%$ son afectadas por conflicto de uso del suelo medio y alto, lo cual evidencia el importante impacto ambiental negativo debido al inadecuado uso del suelo. La similitud entre los porcentajes de afectación para áreas y longitudes de corrientes, como se aprecia en la Tabla 1 , sugiere que las actividades presentes en el corredor están llegando a la corriente hídrica en casi la totalidad de los casos; situación causada por el afán de los propietarios de las tierras, de aprovechar al máximo el área de suelo para su beneficio económico.

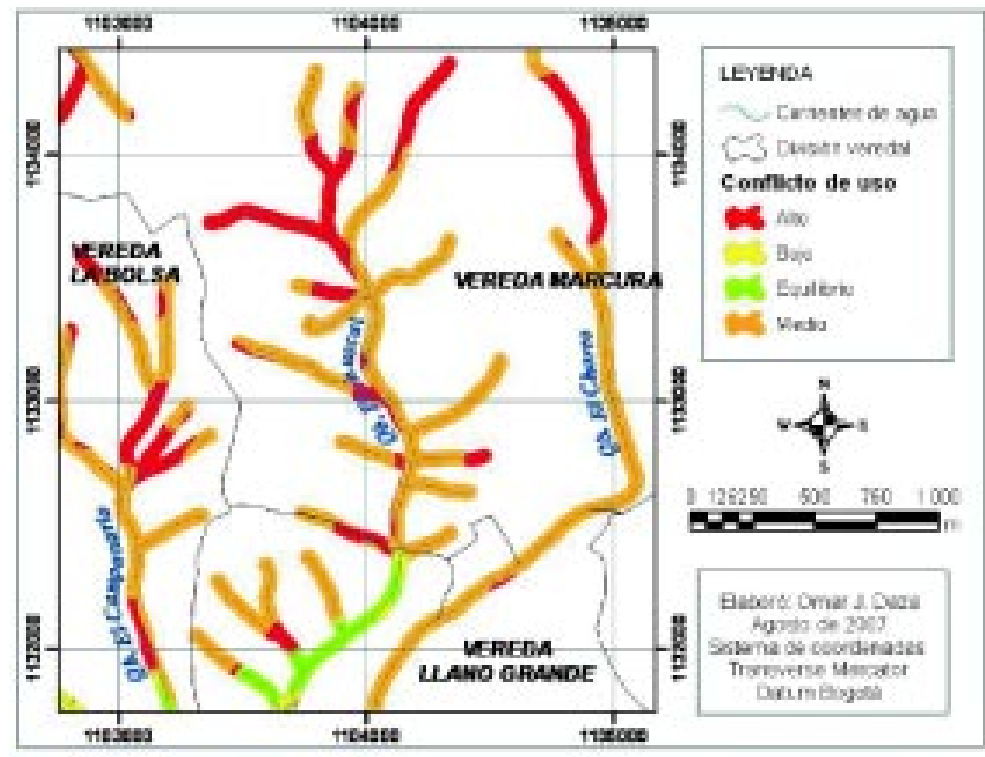

Figura 3. Mapa de conflictividad de uso del suelo en rondas hídricas. 
Un análisis comparativo de dos de las corrientes más importantes del municipio, presenta condiciones diferentes de conflictividad en su uso del suelo. Para el caso de la quebrada El Rosal, un $20 \%$ del área de su ronda, definida para conservación y recuperación, está siendo actualmente utilizada en actividades agropecuarias (conflicto de uso del suelo alto). Mientras que para el Río Chicamocha, aunque no hay presencia de conflicto alto, se presenta la invasión de construcciones en la ronda hídrica. En ambos casos, aunque por diferente causa, se deben tomar medidas para prevenir impactos ambientales irreparables; en el primero, las medidas deben estar orientadas hacia el cambio de uso del suelo y reforestación, y en el segundo, hacia la ubicación de cercados sobre el límite aún sin construir de la ronda del río, para evitar que las construcciones invadan esta área.

Tabla 1. Áreas de conflicto de uso del suelo en rondas hídricas y longitudes de corriente por conflicto

\begin{tabular}{|c|c|c|c|c|}
\hline Tipo de conflicto & Área (ha) & $\begin{array}{l}\text { Porcentaje con } \\
\text { respecto al área } \\
\text { total de ronda }\end{array}$ & L ongitud (m) & $\begin{array}{l}\text { Porcentaje con res- } \\
\text { pecto a la longitud } \\
\text { total de corrientes }\end{array}$ \\
\hline \multicolumn{5}{|l|}{ M unicipio de Paipa } \\
\hline Crecimiento urbano & 26 & 1 & 2,282 & 1 \\
\hline Alto & 703 & 20 & 116.408 & 20 \\
\hline Medio & 1.629 & 48 & 280.973 & 48 \\
\hline Bajo & 520 & 15 & 90.408 & 15 \\
\hline Equilibrio & 542 & 16 & 95.190 & 16 \\
\hline Total & 3.420 & 100 & 585.261 & 100 \\
\hline \multicolumn{5}{|l|}{ Q uebrada el R osal } \\
\hline Crecimiento urbano & 5 & 12 & 768 & 12 \\
\hline Alto & 8 & 20 & 1.352 & 20 \\
\hline Medio & 14 & 35 & 2.154 & 33 \\
\hline Bajo & 10 & 25 & 1.706 & 26 \\
\hline Equilibrio & 3 & 8 & 575 & 9 \\
\hline Total & 40 & 100 & 6.554 & 100 \\
\hline \multicolumn{5}{|l|}{ Río Chicamocha } \\
\hline Crecimiento urbano & 37 & 41 & 6.514 & 43 \\
\hline Alto & 0 & 0 & 0 & 0 \\
\hline Medio & 7 & 8 & 510 & 3 \\
\hline Bajo & 1 & 1 & 139 & 1 \\
\hline Equilibrio & 45 & 50 & 8.106 & 53 \\
\hline Total & 90 & 100 & 15.268 & 100 \\
\hline
\end{tabular}

Fuente: elaboración propia. 
Al comparar el indicador Grado de Conflicto de Uso del suelo en la figura 4 , se encuentra que las veredas cuyas rondas presentan una situación crítica, se sitúan en la parte central del municipio, donde el uso del suelo es principalmente ganadería extensiva y agricultura tradicional.

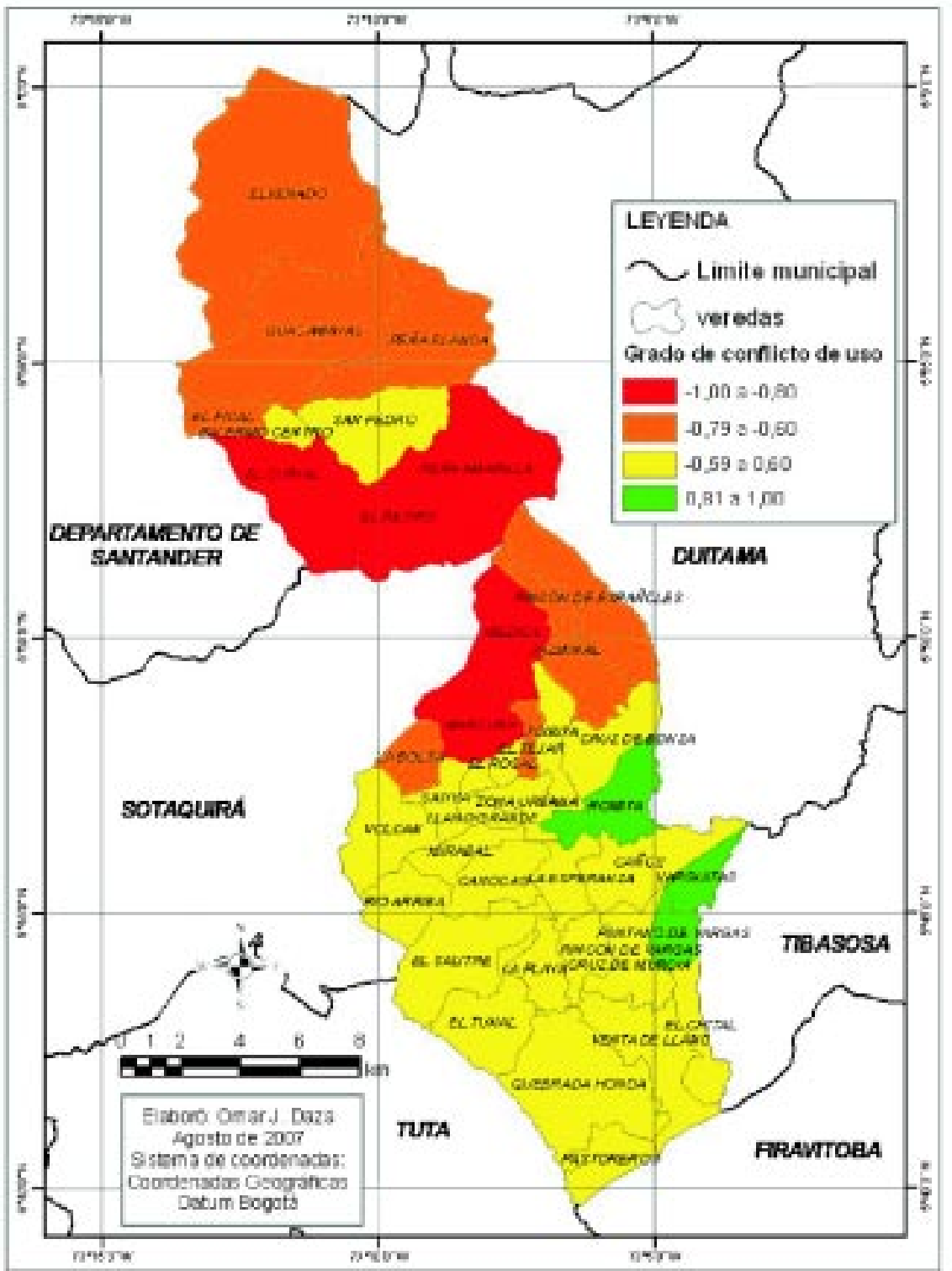

Figura 4. Grado de conflicto de uso del suelo para las veredas de Paipa. 
Las veredas Medios, Marcura, Peña Amarilla, El Retiro y El Curial, cuyo indicador es menor a $-0,8$, presentan la situación más desfavorable, con más del 90\% de las rondas en estado crítico de degradación, causado por conflictos de uso alto, medio y el crecimiento urbano. Las primeras acciones para el cambio de uso del suelo actual deben orientarse hacia las corrientes dentro de estas veredas. Los valores del indicador entre $-0,79$ y $-0,60$ clasifican 8 veredas del municipio, en las cuales la presencia de conflictos con consecuencias perjudiciales está entre $89 \%$ y $80 \%$. Los valores del indicador entre $-0,59$ y 0,6 agrupan a 23 veredas en condiciones de conflictividad intermedia en sus rondas (entre $79 \%$ y 20\%). Finalmente, las veredas Romita, Varguitas y Pantano de Vargas presentan conflictos menores a $19 \%$, siendo éstas las que presentan menor incidencia negativa causada por conflictos de uso del suelo.

La clasificación de las veredas, de acuerdo con el indicador, permite observar que los impactos ambientales negativos, causados por los conflictos de uso del suelo, se localizan en la parte norte del municipio; zona que, además, está asociada con áreas de páramo y bosques altoandino, ecosistemas de gran importancia por sus funciones de regulación hídrica y que, por lo tanto, requieren de medidas urgentes para el cambio de su uso actual e iniciar actividades de reforestación y recuperación.

\section{Conclusiones y comentarios}

A partir de información secundaría proveniente de los planes de ordenamiento territorial y la metodología propuesta, se pudo mostrar la alta afectación, por usos inadecuados del suelo, presente en las rondas hídricas del municipio de Paipa. Se identificaron y localizaron las áreas con conflictos de uso del suelo alto y medio, y se clasificaron las veredas de acuerdo con la conflictividad. El ejercicio realizado aporta, con los resultados obtenidos, en la toma de decisiones sobre cuáles corrientes hídricas se deben intervenir inicialmente, al estar siendo afectadas por conflictos de uso del suelo considerables.

La similitud en los resultados del análisis de área de ronda con conflictos de uso del suelo frente a longitud de corriente afectada, permite concluir que en las áreas de alto y medio conflicto, no existe ningún remanente de vegetación natural del cual se pueda partir para recuperar las condiciones iniciales de la ronda. Además, sugiere que la contaminación causada por las actividades agropecuarias predominantes en la zona, afecta directamente el curso de agua.

El análisis de las rondas hídricas con presencia de alta conflictividad de uso del suelo, permite identificar áreas de intervención prioritaria, bajo la suposición que a mayor conflicto de uso, mayor degradación ambiental (Naizaque, 2001). Pero, no se debe dejar de lado la redefinición del uso del suelo en zonas de conflictividad baja y en equilibrio, ya que, de igual forma, se realizan actividades agropecuarias que 
conllevan graves problemas de contaminación y erosión (Redal et al., 2005), por lo cual, aunque su intervención no es prioritaria, no quiere decir que su uso actual deba ser admitido. No se debe olvidar que todo cambio de uso, que hubiese implicado la eliminación de cobertura natural de los corredores hídricos, afecta la calidad y cantidad del recurso (Wender, 1999).

Los resultados arrojados por la implementación de la metodología en el municipio de Paipa, se vieron limitados espacialmente por los límites políticoadministrativos municipales; en la red hidrográfica empleada, se presentan cortes de corrientes naturales de agua que no corresponden con la realidad. Es por esto que se sugiere, que la metodología propuesta sea empleada para zonas de estudio definidas por los límites de las cuencas hidrográficas; caso para el cual, en la zonificación de conflictos de uso del suelo, puede tomarse del componente de zonificación agroecológica de los planes de ordenación y manejo de las cuencas hidrográficas (Instituto de Hidrología, Meteorología y Estudios Ambientales, 2006). Adicionalmente, estudios posteriores pueden tener en cuenta una posible variación del ancho de la ronda, dependiendo el orden de la corriente dentro de la cuenca (Bentrup y Kellerman, 2004), para dar un ancho de corredor mayor a corrientes ubicadas en zonas altas de la cuenca y evitar los impactos ambientales negativos en los nacimientos y corrientes vulnerables a la reducción o eliminación del agua por su bajo caudal.

De otro lado, ya que dentro de la metodología propuesta en este artículo no se considera la verificación en campo de los resultados obtenidos, se hace necesario, para posteriores proyectos, considerar una etapa que integre la metodología, la planeación y realización de muestreos en campo, para legitimar los resultados obtenidos. 


\section{Literatura citada}

Bentrup G. y Kellerman T. 2004. Where should buffers go? M odeling riparian habitat connectivity in northeast Kansas. J ournal of Soil and Water Conservation, 59:209-215.

Corpoboyacá e Hidrogeología y Geotecnia Ambiental Ltda. 2004. Plan de Manejo, Acción e Inversión para la aplicación de los recursos provenientes del sector eléctrico por las Plantas de generación eléctrica I, II ý III de la Empresa de Energía de Boyacá y la Compañía Eléctrica de Sochagota - Termopaipa IV. Tunja.

Department of Biological and Agricultural Engineering; Riparian Buffers, Stream Notes, Vol. 1. No. 3. Available from internet: www.bae.ncsu.edu/programs/ extension/wqg/sri / riparian5.pdf. Verified: 09/07/2007.

Environmental Systems Research Institute. 2004. Software ArcGIS ArcView 9.0. ESRI, Inc. Redlands, California, USA.

Gonzáles, E. 2005. Erosión: la importancia de la conservación del suelo, Córdoba (España). Córdoba: Asociación Española Agricultura de Conservación suelos vivos.

Instituto de Hidrología, Meteorología y Estudios Ambientales. 2006. Guía técnico- científica para la ordenación y manejo de cuencas hidrográficas en Colombia. Bogotá, D.C.

Instituto Geográfico Agustín Codazzi, IGAC. 2000. Plan Básico de Ordenamiento Territorial de Paipa. Paipa.

IGAC y Corpoica. 2002. Zonificación de los conflictos de uso de las tierras en Colombia. Bogotá, D.C.

Jarro E. 2004. Guía Técnica para la restauración de áreas de ronda y nacederos del distrito capital. Bogotá, D.C.: DAMA.

Lamoine D., Evans J. and Smith K. 2006. A landscape-level Geographic Information System (GIS). Analysis of Streamside M anagament Zones on the Cumberland Plateu. J ournal of Forestry, 104:125-131.

Naizaque J. 2001. Esquema de Ordenamiento Territorial de Cómbita. Cómbita.

Presidencia de la República de Colombia. 1974. Decreto 2811: Por el cual se dicta el Código Nacional de Recursos Naturales Renovables y de Protección del Medio Ambiental. Bogotá, D.C. 
1998. Decreto 1504: Por el cual se reglamenta el manejo del espacio público en los planes de ordenamiento territorial. Bogotá, D.C.

Redal L., Carrasco I. y Gordillo A. 2005. Influencia de los usos del suelo sobre la calidad en la cuenca alta del río Peñas Blancas y estudio de alternativas de desarrollo rural más sostenibles. Costa Rica: Centro Científico Tropical.

Sanabria R. 1999. Generalidades sobre los Sistemas de Información Geográfica: Guía para el curso. Santafé de Bogotá: Universidad Católica de Colombia.

Wender S. 1999. A review of scientific literature of riparian buffers width, extend and vegetation. Georgia: U niversity of Georgia.

Williams T.; Lipscomb D.; English W. and Nickel C. 2003. M apping variable - width streamside management zones for water quality protection. Biomass \& Bioenergy, 24:329-336.

Fecha de recepción: 15 de febrero de 2008

Fecha de aprobación: 25 de agosto de 2008 